\section{GRP-143 POTASSIUM MONITORING: DO WE GIVE IT THE ATTENTION IT DESERVES?}

doi:10.1136/ejhpharm-2013-000276.143

T Roldan Sevilla, E Villamañan Bueno, M Ruano Encinar, C Rueda Perez, Y Larrubia Marfil, E Perez Andreu, T Perez Robles, A Herrero Ambrosio. Hospital Universitario La Paz, Hospital Pharmacy, Madrid, Spain

Background Potassium $(\mathrm{K}+)$ is the principal intracellular cation and is essential to maintain the function of multiple organs. It is a critical component of cardiac conduction and has a narrow therapeutic/toxic range.

Purpose To investigate the effect of pharmaceutical intervention through computerised prescription order entry (CPOE) in hospitalised patients with $\mathrm{K}+$ disorders.

Materials and Methods Prospective study carried out over 7 weeks. Pharmacists first added information about drugs that affect the $\mathrm{K}+$ level as a support in the prescription programme. We then identified patients with abnormal $\mathrm{K}+$ levels using a link with laboratory data $(<3.1$ and $>5.3 \mathrm{mmol} / \mathrm{l})$. Pharmacists reviewed the pharmacotherapy daily in order to detect possible medication errors related to $\mathrm{K}+$ disorders. Lastly we analysed the effect of pharmaceutical recommendations and physician acceptance rate.

Results 183 patients were included (67 \pm 17 years old on average), 128 patients (69.9\%) with hypokalaemia and 55 (30.1\%) with hyperkalaemia. A total of 3,380 electronic prescriptions were selected. Of them, 540 (16.0\%) could affect $\mathrm{K}+$ levels mainly through furosemide, piperacillin-tazobactam and meropenem; pharmacists checked 383 orders thoroughly to prevent possible medication errors. $232(60.6 \%)$ required pharmaceutical recommendations, 130 of them (56.0\%) were related to optimising K+ therapy in hypokalaemic patients and $35(15.0 \%)$ were safety recommendations for closer monitoring. Clinicians accepted $72.4 \%$ of recommendations.

Conclusions There is a high rate of prescription errors related to $\mathrm{K}+$ disorders that could jeopardise patient safety. Pharmaceutical intervention through CPOE helps to minimise them and increases physician awareness of the necessity of closer $\mathrm{K}+$ monitoring in these patients.

No conflict of interest.

\section{GRP-144 POTENTIAL DRUG-DRUG INTERACTIONS IN PATIENTS ADMITTED TO A TRAUMA HOSPITAL}

doi:10.1136/ejhpharm-2013-000276.144

C González-Guerrero, C Salazar Valdebenito, MJ de Dios García, L Girona Brumos, P Lalueza Broto. Hospital de la Vall d'Hebron, Pharmacy, Barcelona, Spain

Background The current complexity of pharmacotherapy in trauma patients increases the risk of drug-drug interactions (DDIs). Purpose To identify potential DDIs (severe/moderate) and their clinical relevance in patients admitted to a tertiary trauma hospital on an ordinary day.

Materials and Methods One-day retrospective observational study performed in patients admitted to a trauma hospital. The following variables were recorded for each patient from the pharmacy database: sex, age and pharmacology treatment during a day of hospitalisation.

A Spanish DDI database (Medinteract NR) was used to determine potential DDIs.

Results The study included 110 patients (54 men and 56 women) with a mean age of 61 years (range 13-94), 45\% of those patients being over 65 years old. The mean number of drugs prescribed per patient was 8.8. We detected 357 potential DDIs (30 severe, 327 moderate) in 89 of the 110 patients (mean potential DDI of 3.5 per patient).
Among the severe potential interactions we highlighted the following risks:

- $20 \%$ involved an increase in the risk of haemorrhage (enoxaparin-acetylsalicylic acid, enoxaparin-acenocoumarol),

- $23 \%$ involved a prolonged OT interval (quetiapinehaloperidol and quetiapine-citalopram),

- $37 \%$ involved a serotoninergic syndrome (due to the association of an opioid analgesic with a selective serotonin reuptake inhibitor)

- $6 \%$ involved rhabdomyolysis: simvastatin-risperidone, simvastatin-amlodipine

Conclusions Due to the high incidence of potential DDIs, the pharmacist should play two key roles when facing a potential interaction: if possible, suggest an alternative with the same therapeutic profile, but without the interaction risk; or evaluate the benefit/risk balance and if it is worth taking the risk, monitor the patient closely and warn the rest of the medical staff.

No conflict of interest.

\section{GRP-145 POTENTIALLY INAPPROPRIATE PRESCRIBING IN ELDERLY FALLERS: A REVIEW OF CURRENT PRACTISE AND THE IMPACT OF PHARMACIST-LED MEDICINES REVIEWS}

doi:10.1136/ejhpharm-2013-000276.145

D O'Sullivan, J Carroll, C Meegan. Mater Misericordiae University Hospital, Pharmacy, Dublin 7, Ireland (Rep.)

Background Falls in the elderly represent a significant health burden in Ireland and internationally. Falls are consistently the most frequently reported patient incident in the MMU Hospital. Polypharmacy and inappropriate prescribing are considered important extrinsic risk factors for falling. Section $\mathrm{H}$ of the Screening Tool of Older Persons' Potentially Inappropriate Prescriptions (STOPP) criteria relates to medicines known to adversely affect fallers.

Purpose To explore the current level of medicines review afforded to elderly fallers.

To determine if use of the STOPP criteria \& subsequent pharmacist intervention result in a greater reduction in the number of prescriptions for, \& the number of patients receiving, $\geq 1$ potentially inappropriate medicines (PIMs), compared to the current standard of care.

To develop prescribing guidelines for inclusion in a future falls prevention policy for the hospital.

Materials and Methods A STOPP-based research protocol for the identification of PIMs in elderly fallers was developed and piloted.

A programme of usual care versus pharmacist intervention was undertaken.

In the intervention study the investigator alerted medical teams to PIM(s) through communication in patients' medical notes.

Ward pharmacists reviewed patients' drug charts after the investigator's review, \& recorded changes to identified PIMs.

The investigator participated in a falls working group set up to develop and implement a policy on the prevention of falls in MMUH.

Results Pharmacist intervention resulted in reductions in both the number of patients prescribed $\geq 1$ PIM, \& prescriptions for PIMs; however, these results failed to reach statistical significance $(\mathrm{P}=0.08 \& 0.074$, respectively).

Patients prescribed $\geq 1$ PIM were taking 3 more regular medicines than those who were not $(p<0.001)$.

The MMUH Falls Working Group developed a policy on falls prevention. The STOPP criteria were used to formulate the relevant sections relating to medicines \& falls.

Conclusions Pharmacist intervention may have a positive impact on rates of potentially inappropriate prescribing in elderly patients who fall during admission. However, further research is needed.

No conflict of interest. 